\title{
Comparative evaluation of paranasal sinus volumes between maxillary deficiency requiring Le Fort osteotomy patients and control patients
}

\author{
Poyzan BOZKURT ${ }^{1}$, Kaan Orhan $^{1}$, and Çağll VURAL ${ }^{1}$ \\ ${ }^{1}$ Ankara University Faculty of Dentistry
}

April 15, 2021

\begin{abstract}
Introduction: The anatomy of the paranasal sinuses is important for many surgeon groups. The precise knowledge of such structures with variable anatomy will be important for the preservation of these structures and the management of complications in surgeries such as endoscopic sinus surgery and osteotomies involving the maxilla. Objective: The purpose of the present study is to investigate volumetric differences between ethmoid, sphenoid and maxillary sinus volumes in patients with maxillary deficiency requiring Le Fort osteotomy and healthy patients, by employing computed tomography imaging. Methods: Computed tomography scans of 120 patients (59 maxillary deficiency patients and 61 control patients) were included in the study. Images were processed, the paranasal sinuses were sculpted out from 3D images and measured. All measurements were taken twice by the same observers. The observers performed the study twice with an interval of 2 weeks to detect intra-observer variability. Results: Ethmoid and left and right maxillary sinus volumes were smaller in the Le Fort group, although no differences were observed for sphenoid sinus volumes. Conclusion: Paranasal sinus volumes varied between maxillary deficiency patients and control patients. This condition may be crucial for the surgeon operating in these areas and should be taken into consideration during surgeries.
\end{abstract}

\section{Introduction}

The intrauterine development of paranasal sinuses commences in the form of mucosal invaginations in the 3rd and 4th months. ${ }^{1}$ There are four paranasal sinuses named after the bone they are associated with: maxillary, ethmoid, sphenoid, and frontal. The ethmoid sinuses are between the orbits. They are formed as labyrinths and vary in number from 3 to 18. The maxillary sinuses are the largest of the paranasal sinuses, located bilaterally in the maxilla. The frontal sinuses are in the frontal bone superior to the orbits and vary in size. The sphenoidal sinuses are in the body of the sphenoid bone and open into the posterior wall of the sphenoid-ethmoidal recess. ${ }^{2}$

The maxillomandibular complex is often affected by developmental problems and growth problems, and unilateral, bilateral, horizontal, vertical and / or transverse deficiencies are observed. Treatment of this complex is often performed by surgical intervention involving one or both jaws. In surgeries involving the maxilla, maxillary sinuses are affected by the surgery and affect the course of surgery and precise knowledge of such structures with variable anatomy will be important. ${ }^{3,4}$ Paranasal sinuses are also very important structures for closely related endoscopic sinus surgeries, endoscopic skull-base interventions like pituitary adenomas, anterior and middle skull-base meningiomas, craniopharyngiomas, surgical repair of (cerebrospinal fluid) CSF fistulas and osteotomies involving the maxilla such as Le Fort osteotomies. ${ }^{3}$ Detailed preoperative investigation is crucial for patient selection and hence desired outcome. ${ }^{5}$

The present study aims to compare ethmoid, sphenoid and maxillary sinus volumes and the presence of Onodi cells in patients with maxillary deficiency requiring Le Fort osteotomy compared to healthy patients, 
by employing computed tomography imaging. No accessible information could be found on this subject during literature search. The authors believe anatomical knowledge of these structures in patients with maxillary deficiency will influence all branches performing surgeries in these areas.

\section{Material and Methods}

Study was conducted in the Department of Dentomaxillofacial radiology of XXX University, Faculty of XXX , between January-November 2019, with ethical approval number: 08/03 given by the faculty Clinical Research Ethics committee and with ClinicalTrials.gov ID: NCTXXX . The study has been carried out in accordance with The Code of Ethics of the World Medical Association. Computed tomography scans of 120 patients (59 maxillary deficiency patients and 61 control) were included in the study. CT scan indications for the group with maxillary deficiency was surgical preparation for maxillary advancement with Le Fort I osteotomy. The control group had no maxillary deficiency and CT images were obtained for several other indications such as temporomandibular disease. CT scans of patients with a history of trauma in the midline skull base, and with the presence of sinus diseases such as chronic sinusitis, nasal polyps, and tumors were excluded from the study.

Imaging procedures: CT imaging's were performed using various CT scanners (GE Lightspeed 16 slice, GE Medical Systems, Milwaukee WI, US; Siemens Somaton Sensation, 16 slice, Siemens Medical Solutions, Erlangen, Germany) at $120 \mathrm{kVp}$ and $25 \mathrm{~mA}$ with a display matrix of 256X256. Images were processed and measured using 3D Synapse Software (Fujifilm, Tokyo, Japan). The CT data were transferred as DICOM files to the software for measurements. The volumes $\left(\mathrm{mm}^{3}\right)$ were measured as semiautomatic segmentation of the right and left maxillary sinuses, and the ethmoid and sphenoid sinus. For segmentations, the thresholding was limited to a minimum of $1024 \mathrm{HU}$ and a maximum of $526 \mathrm{HU} .{ }^{6}$ The ethmoid and sphenoid sinuses were sculpted out from these 3D images. Semiautomatic segmentation was performed to include the entire paranasal sinus anatomies (Figure 1,2 and 3).

For maxillary sinuses, those were clipped according to the surrounding bone structure and the narrowest area of the ostium between the infundibulum and the processus uncinatus using the software's "edit masks" tool. Then, the connection with the outside air was interrupted by slice using segmentation tools. The "region growing" tool was used to divide the segmentation created by thresholding several objects and remove the floating pixels. The paranasal sinuses then were automatically calculated using the software analysis tool. Presence of Onodi cells were noted.

All measurements were taken twice by two blinded radiologists. The radiologists performed the study twice with an interval of 2 weeks to detect intra-observer variability.

Statistical analysis: The analysis of the data was done in SPSS version 11,5. As descriptive, mean \pm standard deviation and median (minimum - maximum) were given for quantitative variables, and number (percentage) was given for qualitative variables. To assess intra-observer reliability, the Wilcoxon matched pairs signed rank test was used for repeat measurements.

In terms of the quantitative variables, the difference between the categories of the qualitative variable with two categories was examined using Student-t test if normal distribution assumptions were provided, and Mann Whitney $U$ test if not. When the difference between two dependent quantitative variables was to be examined, the Wilcoxon Signed Rank test was used, since normal distribution assumptions were not provided. Chi-square test was used to examine the relationship between two qualitative variables. Statistical significance level was taken as 0,05.

\section{Results}

Intra-observer consistency: Repeated CT measurements indicated no significant intra-observer difference for the first observer $(p>0,05)$. Overall intra-observer consistency was rated at $88.4 \%$ between the two evaluations and measurements. All measurements were found to be highly reproducible for both observers and no significant difference obtained from two measurements of the observers $(\mathrm{p}>0,05)$. Thus, the mean values of all measurements were included in the statistical analysis. 
Differences between the two groups for variables are presented in Table 1, and differences between left and right maxillary sinuses, separately for the two groups are presented in Table 2. Statistically significant results were obtained between groups for ethmoid and maxillary sinus volumes (left and right). Ethmoid and maxillary sinus volumes (left and right) were smaller in the Le Fort group. No statistically significant differences were present for sphenoid sinus volume of two groups. Left and right maxillary sinus volumes did not vary in the total study group. The presence of Onodi cells did not vary between groups.

\section{Discussion}

The nasal cavity and paranasal sinuses (frontal, ethmoid, sphenoid and maxillary sinuses) form an anatomical and functional unit covered by the same mucosa. The drainage of these sinuses is from small and narrow openings into the nasal cavity. ${ }^{3}$ While early on, the aeration of these sinuses was detected by anatomical measurements, cadaver studies and conventional radiography, today detection is performed with CT imaging (Computerized tomography) with evaluation in all sections and provides more accurate data. ${ }^{7}$

Cavalieri method is an effective method used to calculate the volume of anatomical structures. It is used to measure the volumes on 3D radiographs of structures that cannot be isolated. In this method, the object whose volume is to be calculated is divided into sections of the same thickness on the radiograph. In these sections, the area of the relevant object is calculated by using the planimetry or the point counting methods. The total volume is calculated by multiplying the calculated total area with the section thickness. ${ }^{8}$ Although the Cavalieri method has been used frequently for calculating volumes of anatomic structures. Today, different workflows have been determined with newly developed software and the volumes of anatomic structures are now calculated with these software. In the present study the method used by Tretiakow et al. has been employed for volume calculation. ${ }^{9}$ After image acquisition, axial, coronal, and sagittal planes were generated by the software and the segmentation process was navigated and inspected. The "threshold", "scissors", "islands", "level" and "smoothing" tools were used respectively and paranasal sinus volumes were calculated.

The complex structure and pneumatization of the paranasal sinuses have been evaluated in numerous studies in different populations and both patient groups with underlying sinus diseases and non-diseased patients have

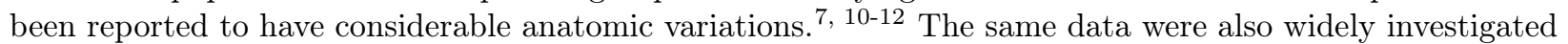
before and after different non-surgical and surgical interventions for various pathologic conditions. ${ }^{4,8,13-18}$ Changes in paranasal sinus morphologies are often varying degrees of hypoplasia, and it has been observed that the formal configurations are commonly preserved. This situation has been reported in genetic syndromes that cause growth center arrests, interval pressure alterations and osteonecrosis. ${ }^{10}$ Shape asymmetries are frequently reported among CLP (cleft lip and palate) human fetuses. Especially the sphenoid sinuses can vary in shape and size. It has been also shown that the maxillary sinuses of CLP fetuses are insufficient in size compared to fetuses without CLP. ${ }^{1}$

Le Fort I osteotomy is one of the techniques among subspecialties of maxillofacial surgery and enables to treat dentofacial deformities of the midface. In this technique, temporary disconnection of the complete maxilla from the midface is required. While it enables to move the maxilla in three dimensions superior repositioning of the maxilla is typically necessary in the maxillofacial deformity treatment with a vertical maxillary excess, such as cases of long face, open bite, or mentum protrusion. ${ }^{8}$ Studies investigating the anatomy of paranasal sinuses before and after Le Fort I osteotomy are great in number. It has been reported that the impact of Le Fort I osteotomy on the upper airway spaces depends on the amount and direction of the skeletal movements, age, gender, and individual variations, and that Le Fort I osteotomy can have an important impact on sinus health. ${ }^{15,16}$ The volume of maxillary sinuses increases compared to that of the pre-surgery and there are reports of possible complications after Le Fort I osteotomy such as causing or aggravating pre-existing maxillary sinus inflammatory processes such as acute sinusitis, the incidence of rhinosinusitis symptoms and iatrogenic damage. ${ }^{13,16,18}$

\section{Conclusion}

In the present study, it was concluded that there were statistically significant differences between ethmoid and 
maxillary sinus volumes of the two groups. Sinus volumes were smaller in the maxillary deficiency group. However sphenoid sinus volume did not vary. The authors believe that this information will be valuable during the operations performed on or around these anatomical structures.

Acknowledgements: None. All authors have no conflict of interest to declare. No financial and material support was obtained for the study.

\section{References}

1. Smith TD, Siegel MI, Mooney MP, Burrows AM, Todhunter JS. Formation and enlargement of the paranasal sinuses in normal and cleft lip and palate human fetuses. Cleft Palate Craniofac J. 1997;34(6):4839 .

2. Henson B, Drake TM, Edens MA. Anatomy, Head and Neck, Nose Sinuses. StatPearls. https://www.ncbi.nlm.nih.gov/books/NBK513272/ Accessed 01 November 2020

3. Perez-Pinas I, Sabate J, Carmona A, Catalina-Herrera CJ, Jimenez-Castellanos J. Anatomical variations in the human paranasal sinus region studied by CT. J Anat. 2000;197(2):221-227.

4. Bin LR, Filho LI, Yamashita AL, de Souza Pinto GN, Mendes RA, Ramos AL, et al. How does bimaxillary orthognathic surgery change dimensions of maxillary sinuses and pharyngeal airway space? Angle Orthod. 2020;90(5):715-722.

5. Mantoni M, Larsen P, Hansen H, Tos M, Berner B, Orntoft S. Coronal CT of the paranasal sinuses before and after functional endoscopic sinus surgery. Eur Radiol. 1996;6(6):920-924.

6. Panou E, Motro M, Ates M, Acar A, Erverdi N. Dimensional changes of maxillary sinuses and pharyngeal airway in Class III patients undergoing bimaxillary orthognathic surgery. Angle Orthod. 2013;83:824-831.

7. Fernandez JS, Escuredo JA, Del Rey AS, Montoya FS. Morphometric study of the paranasal sinuses in normal and pathological conditions. Acta oto-laryngol. 2000;120(2):273-278.

8. Akbulut N, Cakmak ESK, Bayrak S. Assessment of Maxillary Sinus Changes After Le Fort I Osteotomy Surgery. J Craniofac Surg. 2020;31(5):e497-e501.

9. Tretiakow D, Tesch K, Meyer-Szary J, Markiet K, Skorek A. Three-dimensional modeling and automatic analysis of the human nasal cavity and paranasal sinuses using the computational fluid dynamics method. Eur Arch Otorhinolaryngol. 2020;1-11.

10. Lawson W, Patel ZM, Lin FY. The development and pathologic processes that influence maxillary sinus pneumatization. Anat Rec. 2008;291(11):1554-1563.

11. Xu X, Zhang ZY, Li BH, Tang XJ, Yin L, Liu W. Three-Dimensional Measurement of Maxillary Involvement in Hemifacial Microsomia in Children. J Craniofac Surg. 2020;31(2):444-447.

12. Marino MJ, Riley CA, Wu EL, Weinstein JE, Emerson N, McCoul ED. Variability of Paranasal Sinus Pneumatization in the Absence of Sinus Disease. Ochsner J. 2020;20(2):170-175.

13. Moses JJ, Lange CR, Arredondo A. Endoscopic treatment of sinonasal disease in patients who have had orthognathic surgery. Br J Oral Maxillofac Surg. 2000;38(3):177-184.

14. Pamporakis P, Nevzatoğlu Ş, Küçükkeleş N. Three-dimensional alterations in pharyngeal airway and maxillary sinus volumes in Class III maxillary deficiency subjects undergoing orthopedic facemask treatment. Angle Orthod. 2014;84(4):701-707.

15. Almuzian M, Almukhtar A, Ju X, Al-Hiyali A, Benington P, Ayoub A. Effects of Le Fort I osteotomy on the nasopharyngeal airway-6-month follow-up. J Oral Maxil Surg 2016;74(2):380-391.

16. Nocini PF, D'Agostino A, Trevisiol L, Favero V, Pessina M, Procacci P. Is Le Fort I osteotomy associated with maxillary sinusitis? J Oral Maxillofac Surg. 2016;74(2):400-e1.

17. Kurt G, Altug-Ataç AT, Atac MS, Karasu HA. Changes in nasopharyngeal airway following orthopedic and surgically assisted rapid maxillary expansion. J Craniofac Surg. 2010;21(2):312-317.

18. Procacci P, Lanaro L, Trevisiol L, Bertossi D, Zotti F, Fabio L, et al. Is post orthognathic maxillary sinusitis related to sino-nasal anatomical alterations? J Cranio Maxill Surg. 2019;47(6):876-882.

\section{Tables}

Table 1. Differences between the two groups for variables. 


\begin{tabular}{|c|c|c|c|}
\hline Variables & Variables & Maxillary Deficiency Group & Control Group \\
\hline \multirow[t]{2}{*}{ Age } & Mean \pm SD & $27,59 \pm 6,07$ & $29,41 \pm 10,84$ \\
\hline & Median (Min.-Max.) & $27,00(18,00-45,00)$ & $26,00(17,00-69,00)$ \\
\hline \multirow[t]{2}{*}{ Sex, n (\%) } & Male & $34(57,6)$ & $24(39,3)$ \\
\hline & Female & $25(42,4)$ & $37(60,7)$ \\
\hline \multirow[t]{2}{*}{ Ethmoid Sinus Volume $\left(\mathrm{mm}^{3}\right)$} & Mean \pm SD & $10,36 \pm 3,42$ & $13,80 \pm 4,10$ \\
\hline & Median (Min.-Max.) & $10,60(3,11-17,51)$ & $14,40(4,19-25,31)$ \\
\hline \multirow{2}{*}{ Sphenoid Sinus Volume $\left(\mathrm{mm}^{3}\right)$} & Mean \pm SD & $12,21 \pm 4,96$ & $12,33 \pm 4,71$ \\
\hline & Median (Min.-Max.) & $11,63(2,44-27,78)$ & $11,98(4,81-26,48)$ \\
\hline \multirow[t]{2}{*}{ Left Maxillary Sinus Volume $\left(\mathrm{mm}^{3}\right)$} & Mean \pm SD & $14,17 \pm 4,87$ & $16,24 \pm 4,50$ \\
\hline & Median (Min.-Max.) & $14,10(2,48-25,30)$ & $16,09(3,13-27,25)$ \\
\hline \multirow[t]{2}{*}{ Right Maxillary Sinus Volume $\left(\mathrm{mm}^{3}\right)$} & Mean \pm SD & $13,92 \pm 5,27$ & $15,79 \pm 4,24$ \\
\hline & Median (Min.-Max.) & $13,94(3,81-24,88)$ & $15,09(7,10-30,31)$ \\
\hline Onodi cells & Onodi cells & 28 & 21 \\
\hline
\end{tabular}

SD: Standard deviation; a: Student-t test; b: Mann-Whitney U test, c: Chi-square test

Table 2. Differences between left and right maxillary sinuses, separately for the two groups.

\begin{tabular}{lllll}
\hline Group & Maxillary Deficiency Group & p value & Control Group & p value \\
\hline Left Maxillary Sinus Volume $\left.\mathbf{( m m}^{\mathbf{3}}\right)$ & $14,17 \pm 4,87$ & 0,824 & $16,24 \pm 4,50$ & 0,196 \\
& $14,10(2,48-25,30)$ & & $16,09(3,13-27,25)$ & \\
Right Maxillary Sinus Volume $\left(\mathbf{m m}^{\mathbf{3}}\right)$ & $13,92 \pm 5,27$ & & $15,79 \pm 4,24$ & \\
& $13,94(3,81-24,88)$ & & $15,09(7,10-30,31)$ \\
\hline
\end{tabular}

\section{Figure Legends}

Figure 1: View of the Maxillary sinus during automatic calculation using the software analysis tool. (a) Coronal (b) Axial, and (c) Sagittal views.

Figure 2: View of the Sphenoid sinus during automatic calculation using the software analysis tool. (a) Coronal (b) Axial, and (c) Sagittal views.

Figure 3 : View of the Ethmoid sinus during automatic calculation using the software analysis tool. (a) Coronal (b) Axial, and (c) Sagittal views. 


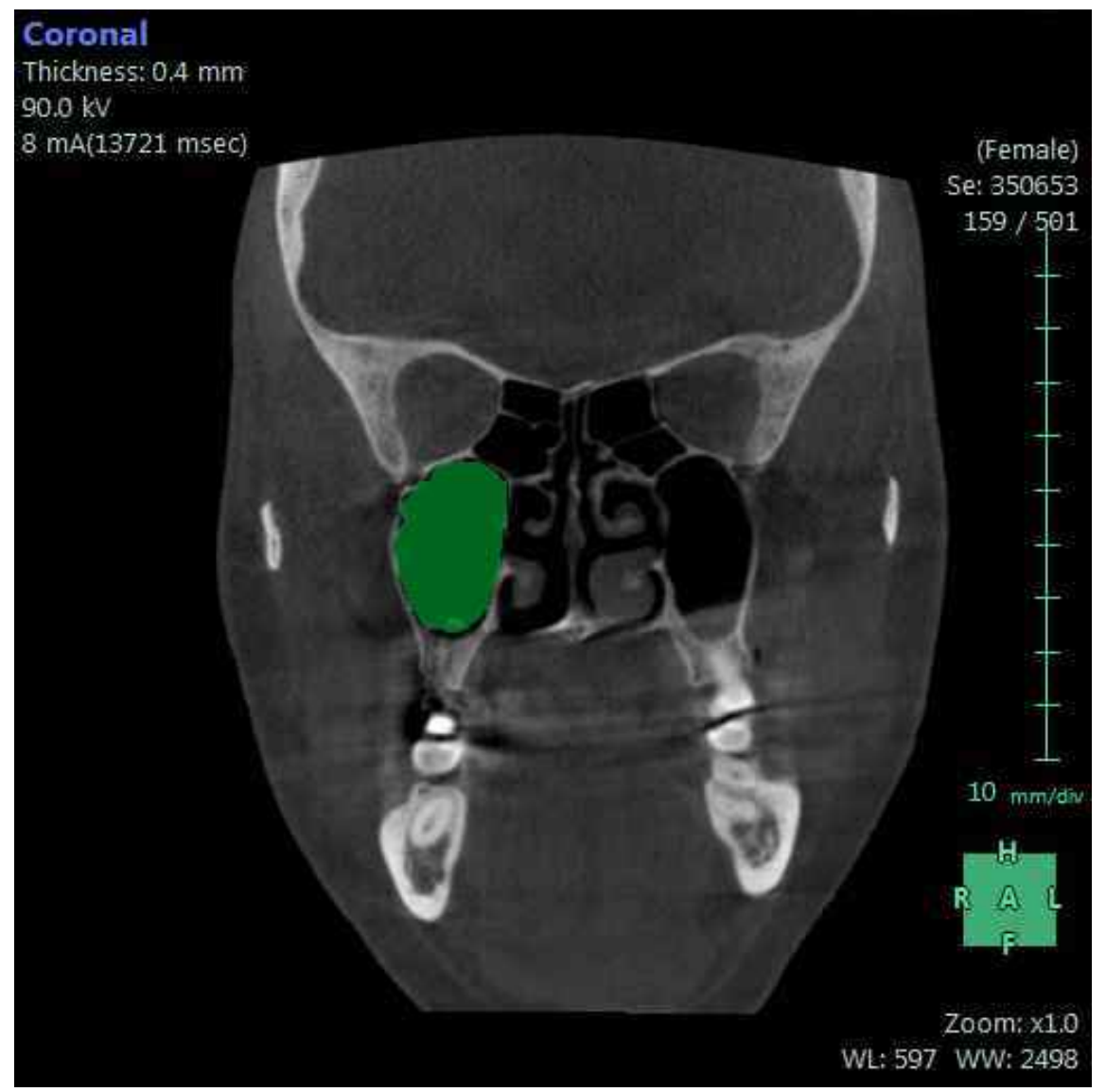




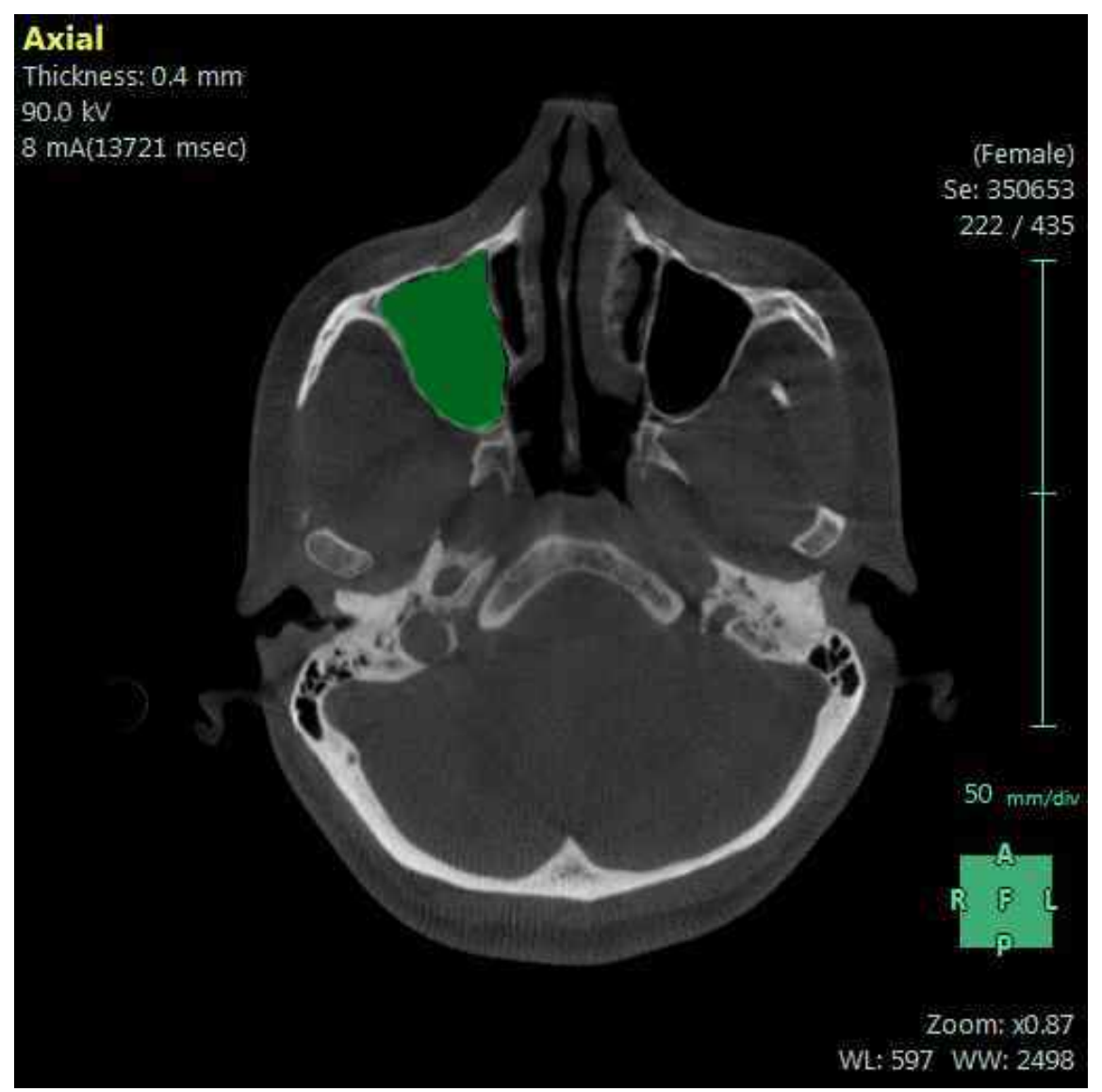




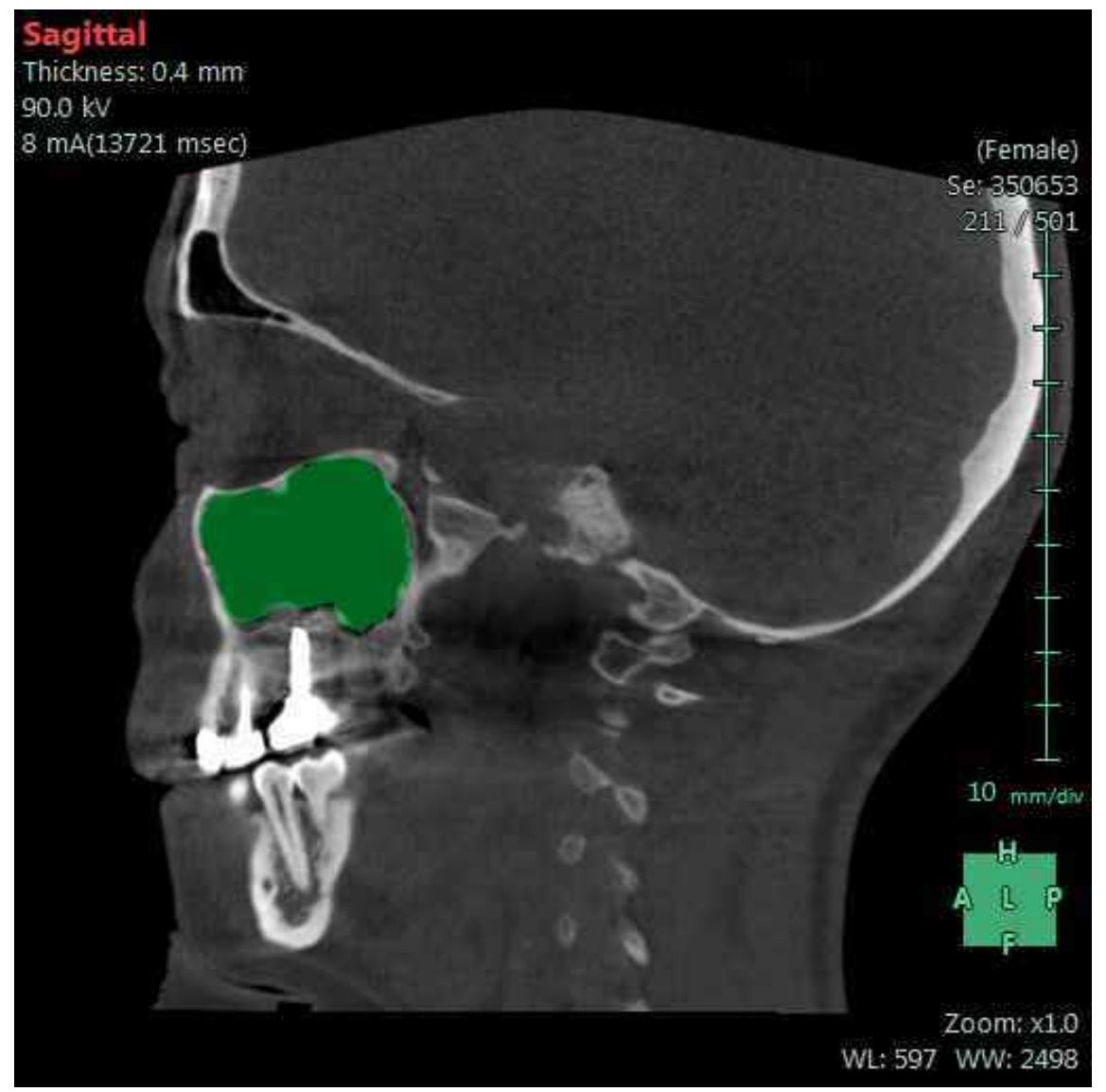




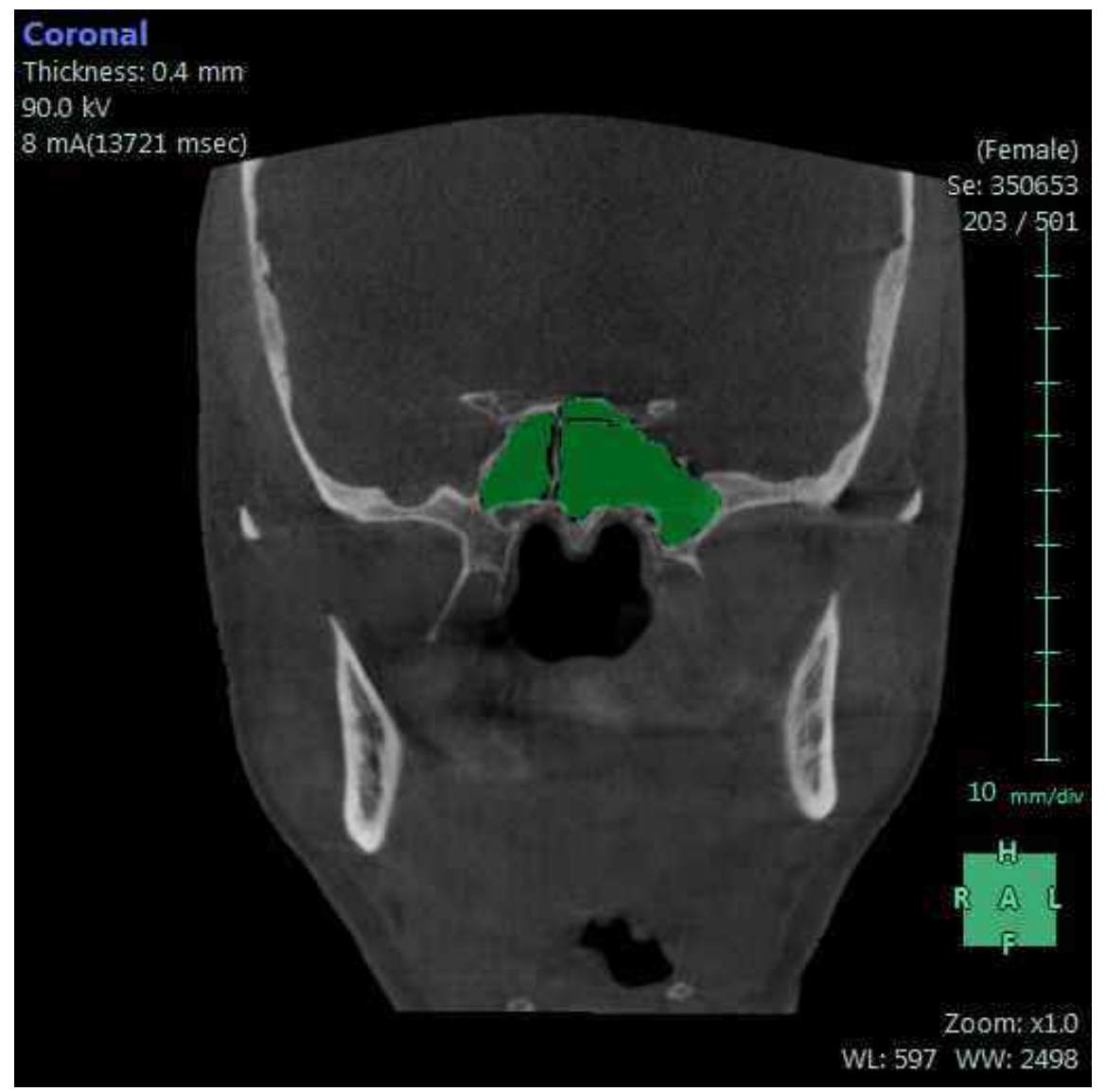




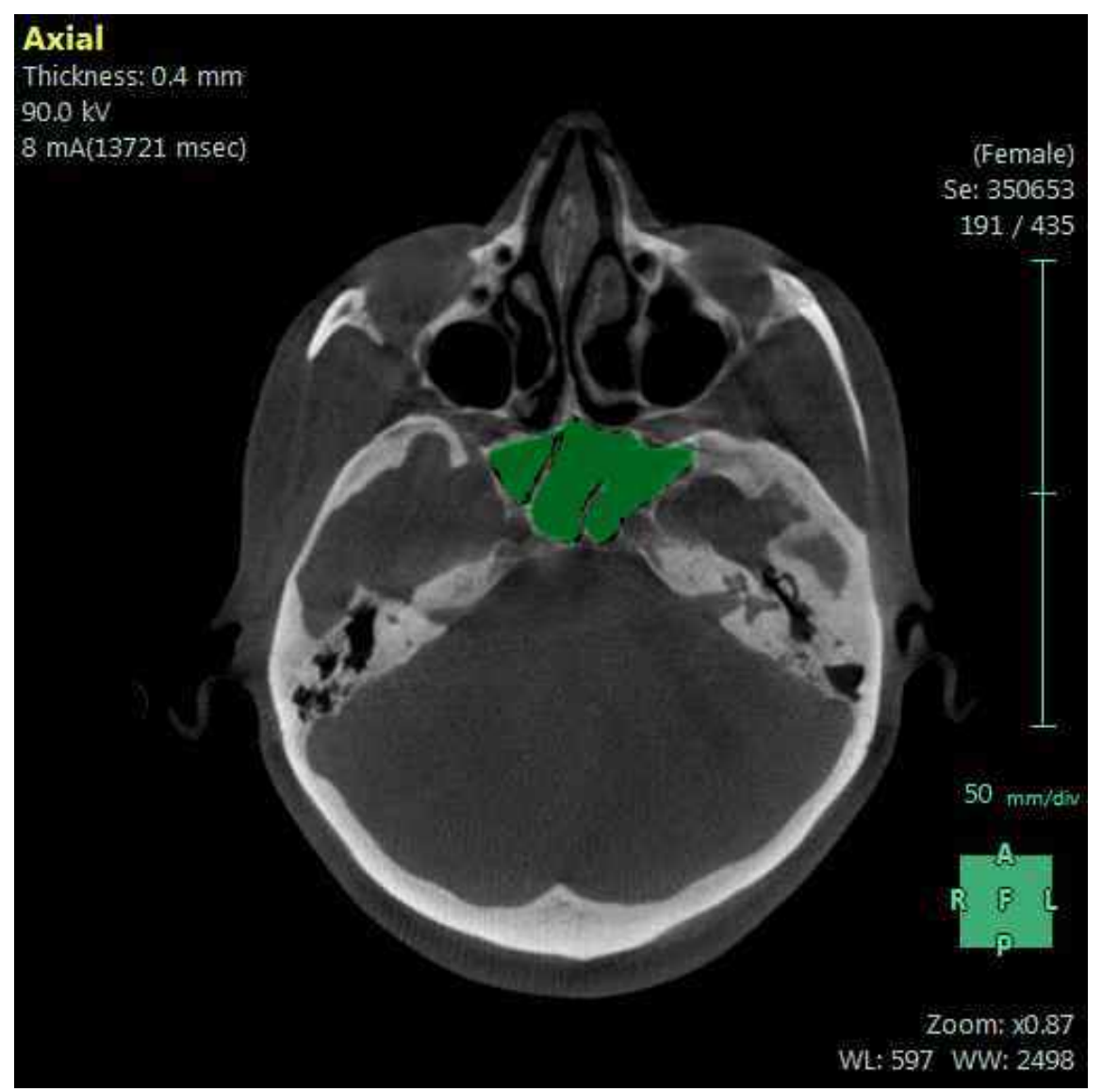




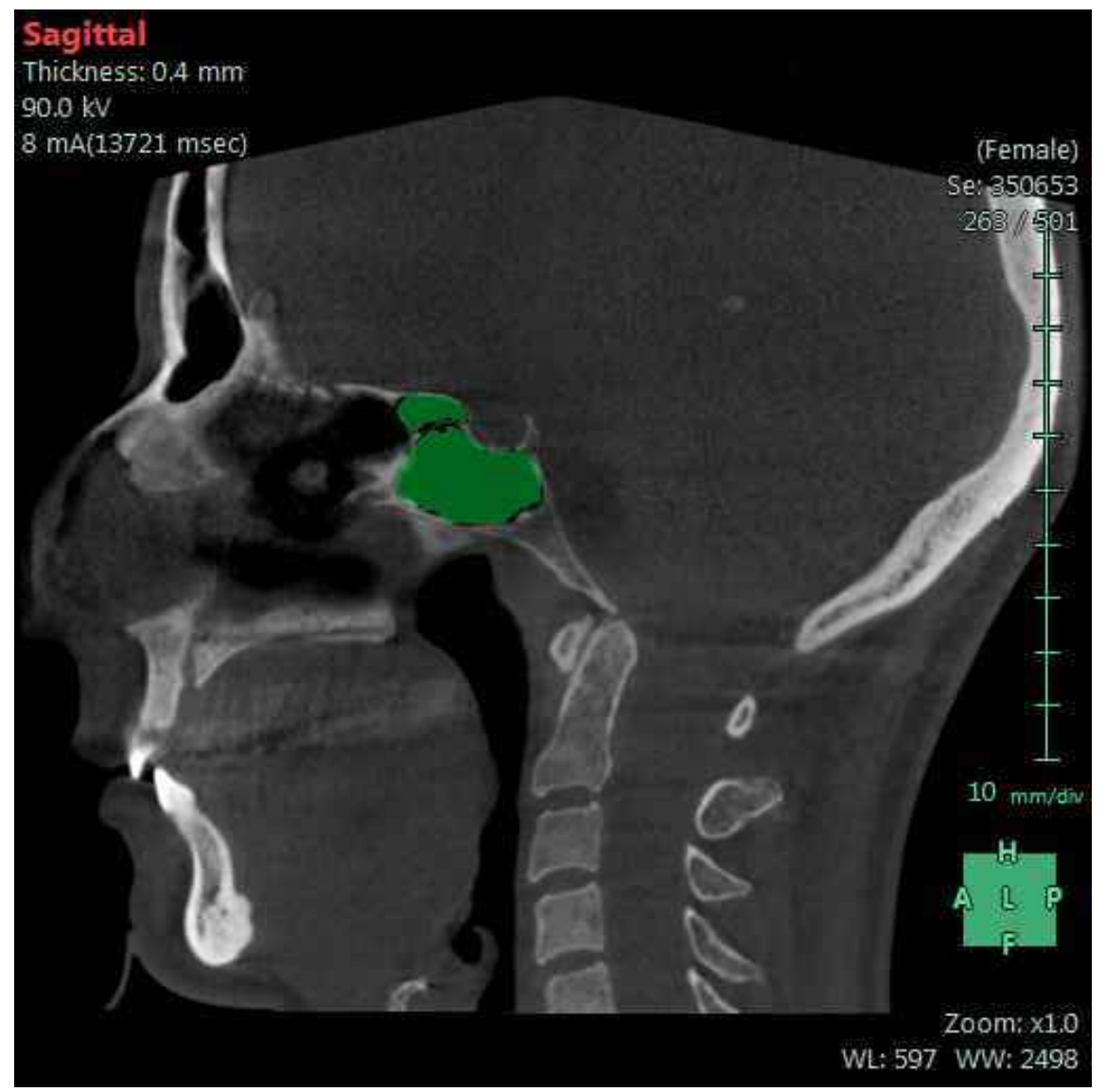




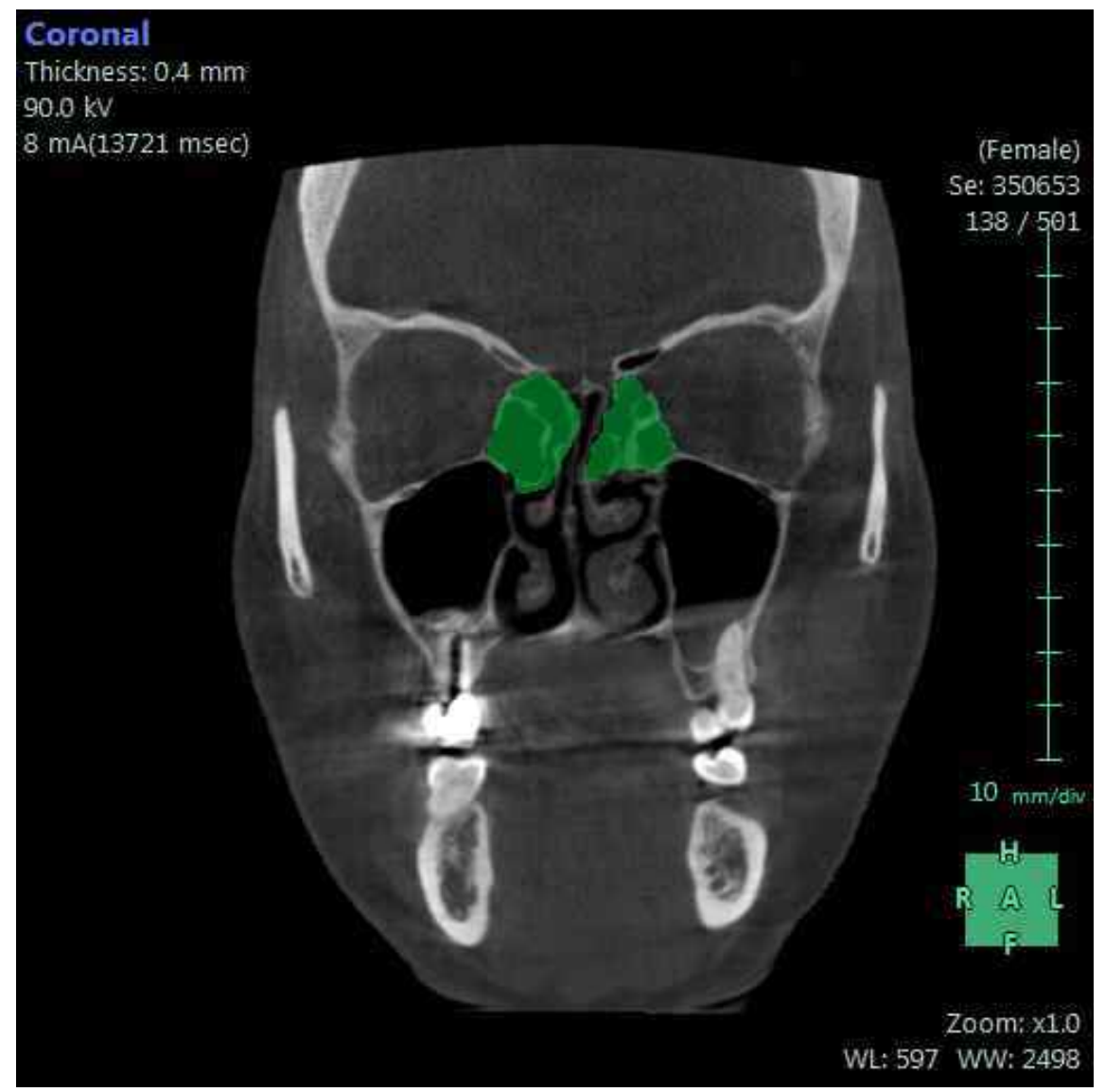




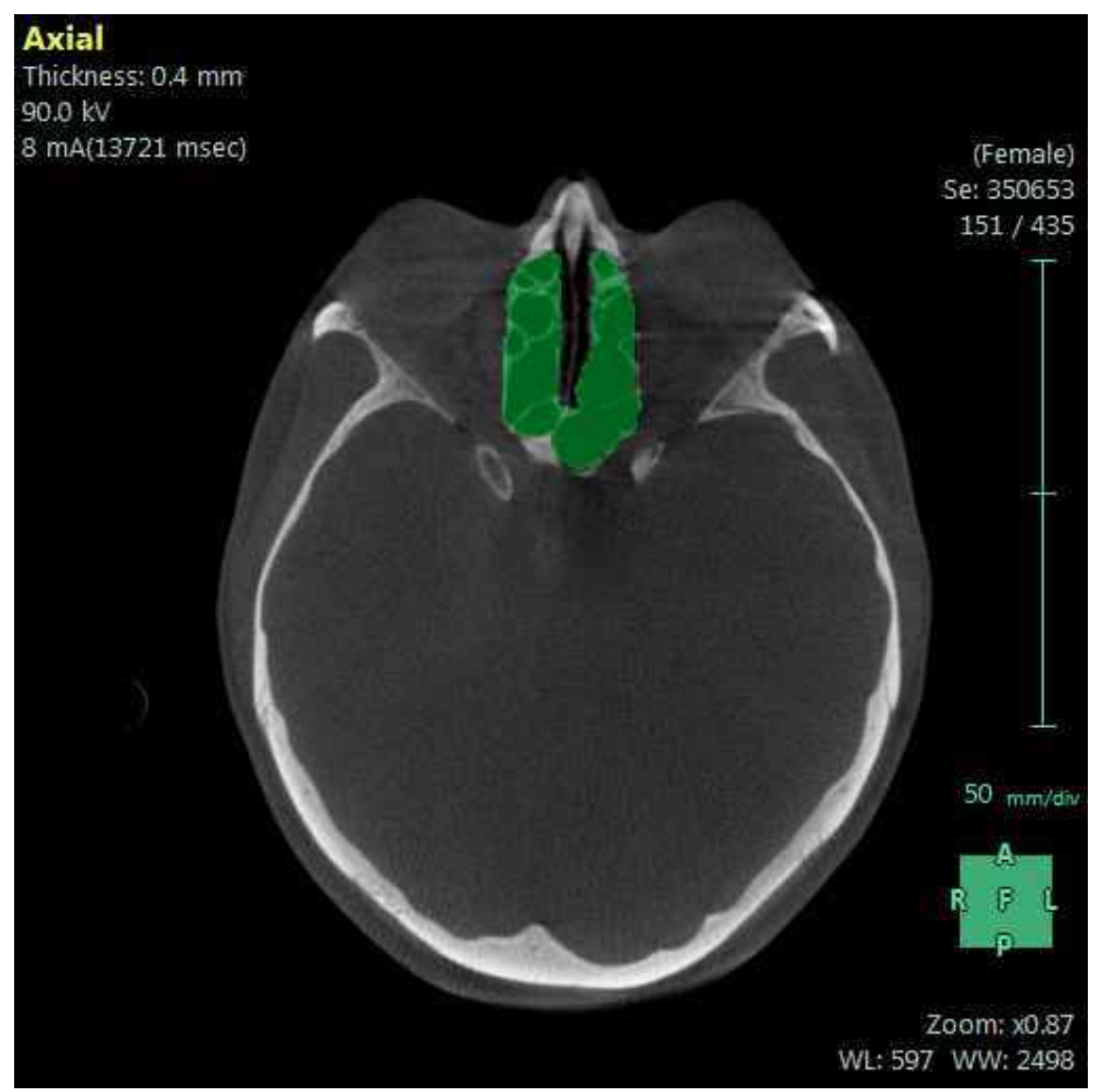




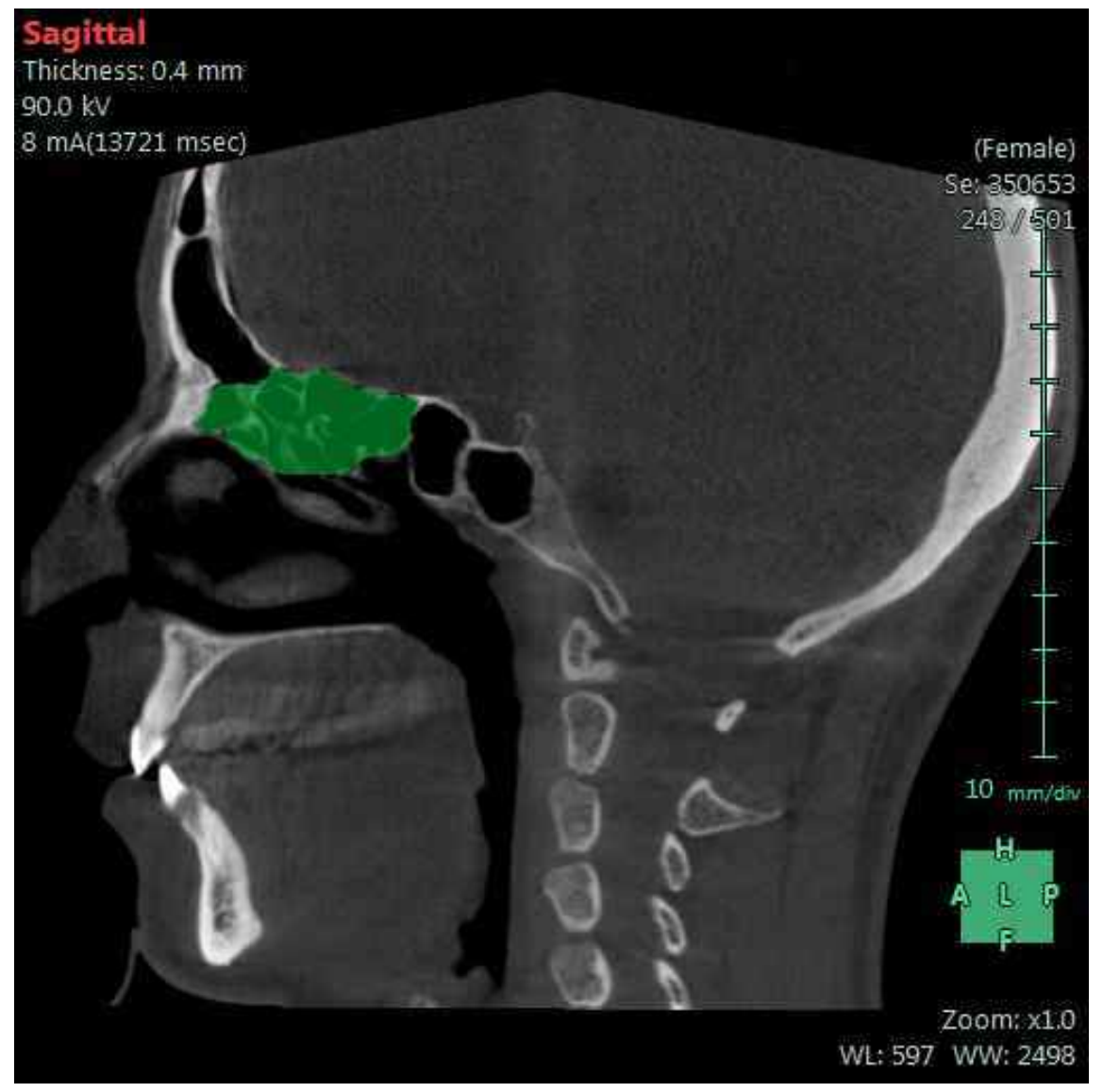

